\title{
Commensal enteric bacteria lipopolysaccharide impairs host defense against disseminated Candida albicans fungal infection
}

\author{
TT Jiang ${ }^{1,2}$, V Chaturvedi ${ }^{1}$, JM Ertelt $^{1}, \mathrm{~L} \mathrm{Xin}^{1}$, DR Clark $^{1}$, JM Kinder ${ }^{1}$ and SS Way ${ }^{1}$
}

Commensal enteric bacteria maintain systemic immune responsiveness that protects against disseminated or localized infection in extra-intestinal tissues caused by pathogenic microbes. However, as shifts in infection susceptibility after commensal bacteria eradication have primarily been probed using viruses, the broader applicability to other pathogen types remains undefined. In sharp contrast to diminished antiviral immunity, we show commensal bacteria eradication bolsters protection against disseminated Candida albicans fungal infection. Enhanced antifungal immunity reflects more robust systemic expansion of Ly6G ${ }^{\text {hi }}$ Ly6 $6 C^{\text {int }}$ neutrophils, and their mobilization into infected tissues among antibiotic-treated compared with commensal bacteria-replete control mice. Reciprocally, depletion of neutrophils from expanded levels or intestinal lipopolysaccharide reconstitution overrides the antifungal protective benefits conferred by commensal bacteria eradication. This discordance in antifungal compared with antiviral immunity highlights intrinsic differences in how commensal bacteria control responsiveness for specific immune cell subsets, because pathogenspecific $\mathrm{CD}^{+}{ }^{+} \mathrm{T}$ cells that protect against viruses were suppressed similarly after $\mathrm{C}$. albicans and influenza $\mathrm{A}$ virus infection. Thus, positive calibration of antiviral immunity by commensal bacteria is counterbalanced by restrained activation of other immune components that confer antifungal immunity.

\section{INTRODUCTION}

Commensal microbes are increasingly recognized to control systemic immune homeostasis and responsiveness. As the greatest density of commensal bacteria reside within the intestinal tract with an estimated 100 trillion organisms comprising over 1,000 individual species, ${ }^{1}$ the symbiotic relationship between commensal microbes and the mammalian host have appropriately been most extensively characterized in this tissue. Accumulating evidence shows enteric commensal bacteria have important roles in maintaining local immune homeostasis, along with digestion and nutrient absorption. ${ }^{1}$ In turn, disrupting the composition of intestinal bacteria represents an important iatrogenic cause of diarrhea from antibiotics used for infection treatment or prevention. ${ }^{2}$ Although the microbial culprits in most cases are unidentified, a classic example is toxin-induced intestinal inflammation from Clostridium difficile overgrowth among antibiotic-exposed individuals. ${ }^{2}$ Disruptions in intestinal microbiota composition similarly occur among individuals with ulcerative colitis or Crohn's disease, ${ }^{3}$ and the communicability of aberrant intestinal inflammation to genetically non-susceptible hosts reinforces the immune-regulatory properties of enteric commensal microbes. ${ }^{4}$ Thus among healthy individuals, commensal intestinal microbes and immune cells sustain a delicate crosstalk that silences pathological inflammation while simultaneously preserving host defense against enteric pathogens.

Given the systemic distribution of intestinal bacteria structural components, ${ }^{5}$ it is remarkable but perhaps not surprising that commensal bacteria have also been shown to regulate systemic inflammation and immune responsiveness in a variety of extra-intestinal tissues. For example, a shift in abundance between intestinal Bacteroidetes and Firmicutes spp. is linked with human obesity, and increased body fat accumulation in animals after transmission of this skewed microbiota further reinforces the causative role for these commensal bacterial shifts. ${ }^{6}$ Similarly, germ-free mice compared with conventionally

${ }^{1}$ Division of Infectious Diseases, Department of Pediatrics, Cincinnati Children's Hospital Medical Center, University of Cincinnati College of Medicine, Cincinnati, Ohio, USA and ${ }^{2}$ Medical Scientist Training Program, University of Cincinnati College of Medicine, Cincinnati, Ohio, USA. Correspondence: SS Way (singsing.way@cchmc.org) 
housed mice on susceptible genetic backgrounds more readily develop autoimmune diabetes, whereas intestinal recolonization with commensal microbes attenuates disease by inducing tolerance to pancreas-specific antigens. ${ }^{7,8}$ Conversely, for other autoimmune disorders such as rheumatoid arthritis or experimental autoimmune encephalomyelitis that stem from pathological Th17 responses, commensal bacteria and in particular, segmented filamentous bacteria, have a detrimental role. ${ }^{9,10}$ Thus, commensal bacteria can potently regulate activation of systemic immune components with beneficial or harmful impacts on autoimmunity in extra-intestinal tissues.

Commensal bacteria have been shown to have equally pivotal roles with regards to susceptibility against more pathogenic microbes. During systemic or extra-intestinal infection caused by viruses, commensal bacteria appear uniformly protective in augmenting host defense. Studies using viral infections to probe immune shifts following antibiotic-induced eradication of commensal bacteria consistently show blunted expansion of protective virus-specific $\mathrm{CD} 8^{+} \mathrm{T}$ cells, along with impaired clearance of influenza $\mathrm{A}$ in the respiratory tract and viremia caused by lymphocytic choriomeningitis virus. ${ }^{11,12}$ These findings may explain the clinical observations of antibioticassociated susceptibility to viral respiratory infection, ${ }^{13}$ and alleviation of viral gastroenteritis by probiotics. ${ }^{14}$ Importantly, however, as viruses, naturally resistant to antibiotics used to eradicate commensal bacteria, have been widely utilized for probing shifts in host defense following commensal bacteria elimination, the extension of these protective benefits to other pathogen types remains undefined. Nonetheless, this represents a critically important question considering the immune components bolstered by commensal microbes that protect against viruses are either dispensable (e.g., $\mathrm{CD} 8^{+} \mathrm{T}$ cells, interferon- $\gamma)^{11,12,15-17}$ or have inconsistent roles (e.g., interferon- $\alpha / \beta)^{11,18,19}$ in antifungal immunity. Accordingly, to investigate how commensal bacteria controls responsiveness of immune components that protect against systemic fungal infection, the impacts of commensal bacteria eradication on susceptibility to intravenous Candida albicans infection were evaluated. Surprisingly, we show eradication of commensal bacteria bolsters antifungal immunity with enhanced systemic neutrophil expansion and enriched accumulation in infected tissues, which is in sharp contrast to diminished resistance against viral pathogens. Thus, our findings establish fundamentally discordant roles for commensal bacteria in regulating antifungal compared with antiviral immunity.

\section{RESULTS}

Depletion of commensal enteric bacteria protects against disseminated $C$. albicans infection

To investigate how commensal intestinal microbiota influences antifungal host defense, susceptibility to $C$. albicans was evaluated in mice administered a combination of antibiotics in the drinking water (ampicillin, gentamicin, metronidazole, neomycin, vancomycin) previously shown to deplete enteric commensal bacteria. ${ }^{11}$ Our initial studies confirmed the validity of this approach as recoverable bacteria under aerobic and anaerobic growth conditions became uniformly eliminated in the feces of antibiotic-treated mice compared with control mice maintained on autoclaved drinking water (Figure 1a). Moreover, these antibiotics collectively had no impact on C. albicans growth in cell-free broth media (Figure 1b), illustrating potential differences in host susceptibility following antibiotic administration reflect shifts in antifungal immunity. Consistent with the restricted pathogen distribution described after intravenous C. albicans inoculation, ${ }^{20,21}$ recoverable fungi were found almost exclusively within the kidneys with only sporadic colonies recovered from other tissues including the brain, liver, and spleen, regardless of antibiotic treatment (Figure 1c,d). Interestingly, while progressively increasing fungal pathogen burden was found for commensal bacteriareplete control mice maintained on autoclaved drinking water through the first 20 days after infection, recoverable C. albicans declined among antibiotic-treated mice beginning day 10 , and fungal pathogen burden was reduced by $>100$-fold compared with control mice by day 20 post infection (Figure 1c). Significantly improved overall survival paralleled these reductions in $C$. albicans pathogen burden in commensal bacteria-eradicated mice (77\% survival among antibiotictreated compared with $47 \%$ survival among control mice, $P<0.05)$ (Figure 1e). Thus, antibiotic-induced eradication of commensal bacteria augments protection against disseminated C. albicans infection.

\section{Systemic expansion and tissue accumulation of Ly6G ${ }^{\text {hi }}$ Ly6C ${ }^{\text {int }}$ neutrophils after commensal bacteria eradication}

Given the pivotal importance of neutrophil mobilization and recruitment in host defense against C. albicans, ${ }^{22,23}$ potential differences in this immune cell subset were evaluated after C. albicans intravenous inoculation among antibiotic-treated compared with control mice. Starting at comparable levels prior to infection, expansion of CD11b ${ }^{+}$Ly $6 G^{\text {hi }}{ }^{2 y} 6 C^{\text {int }}$ neutrophils in the blood and accumulation within the kidneys were both significantly increased beginning 5 days after infection in commensal bacteria-eradicated mice (Figure 2a,b). Higher neutrophil levels were found in the blood and kidneys of antibiotic-treated compared with control mice despite $>100$-fold reduced C. albicans pathogen burden 20 days post infection (Figures $\mathbf{1} \mathbf{c}$ and $\mathbf{2 a}, \mathbf{b}$ ). These quantitative immunecell shifts appear limited to neutrophils as the proportion of Ly $6 \mathrm{G}^{\mathrm{lo}} \mathrm{Ly} 6 \mathrm{C}^{\mathrm{hi}}$ inflammatory monocytes, B cells, as well as $\mathrm{CD}^{+}{ }^{+}$and $\mathrm{CD}^{+}{ }^{+} \mathrm{T}$ cells did not differ significantly among antibiotic-treated compared with control mice (Supplementary Figure S1 online). Enumerating absolute cell numbers further verified Ly $6 \mathrm{G}^{\text {hi }}$ Ly $6 \mathrm{C}^{\text {int }}$ neutrophil expansion was most pronounced compared with other immune cell subsets after intravenous C. albicans infection, regardless of commensal bacteria depletion (Figure 2c). Thus, enhanced protection against $C$. albicans infection after antibiotic-induced eradication of commensal bacteria is associated with enhanced peripheral Ly $6 \mathrm{G}^{\text {hi }} \mathrm{Ly} 6 \mathrm{C}^{\text {int }}$ neutrophil expansion and mobilization of these cells into infected tissue. 

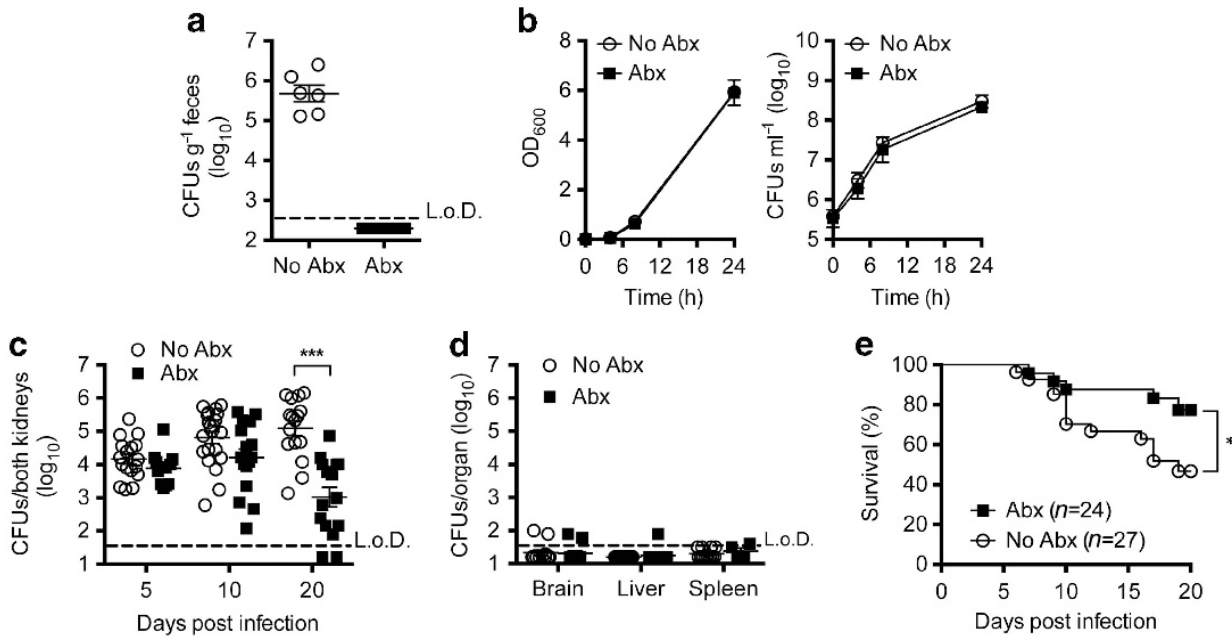

Figure 1 Enhanced protection against disseminated C. albicans infection after antibiotic-induced eradication of commensal bacteria. (a) Fecal colonyforming units (CFUs) after administration of ampicillin $\left(0.5 \mathrm{mg} \mathrm{ml}^{-1}\right)$, gentamicin $\left(0.5 \mathrm{mg} \mathrm{ml}^{-1}\right)$, metronidazole $\left(0.5 \mathrm{mg} \mathrm{ml}^{-1}\right)$, neomycin $\left(0.5 \mathrm{mg} \mathrm{ml}^{-1}\right)$, and vancomycin $\left(0.25 \mathrm{mg} \mathrm{ml}^{-1}\right)$ in the drinking water for 14 days compared with control mice maintained on autoclaved drinking water. (b) $C$. albicans growth kinetics in yeast extract-peptone-adenine-dextrose liquid media without supplementation or supplemented with antibiotics described in a enumerated by optical density (left) or recoverable fungal CFUs after plating serial dilutions onto brain-heart infusion agar plates (right). (c) Recoverable C. albicans CFUs at the indicated time points after intravenous infection with $5 \times 10^{4} \mathrm{C}$. albicans among antibiotic-treated (ampicillin, gentamicin, metronidazole, neomycin, and vancomycin added to the drinking water 14 days prior to infection) compared with control mice maintained on autoclaved drinking water. (d) Recoverable C. albicans CFUs in each tissue day 10 post infection among antibiotic-treated or control mice. (e) Percent survival after intravenous infection with $5 \times 10^{4} \mathrm{C}$. albicans for each group of mice described in $\mathbf{c}$. These data are reflective of at least three independent experiments each with similar results. ${ }^{\star} P<0.05,{ }^{\star * *} P<0.001$. Abx, antibiotic-treated; L.o.D., limit of detection.

Along with enhanced quantitative neutrophil expansion among antibiotic-treated compared with commensal bacteriareplete control mice, potential qualitative shifts in their activation were also investigated. By enriching for neutrophils using negative selection, $\mathrm{Ly} 6 \mathrm{G}^{\text {hi }} \mathrm{Ly} 6 \mathrm{C}^{\text {int }}$ cells could be routinely isolated from the peripheral blood with $\geq 95 \%$ purity (Figure 3a), allowing their antifungal capacity to be compared among each group of C. albicans-infected mice. Despite more accelerated C. albicans clearance among antibiotic-treated mice after infection in vivo (Figure 1c), neutrophils isolated from commensal bacteria-depleted and control mice had comparable fungicidal activity on a per-cell basis at each post-infection time point (Figure 3b). In turn, expression of molecules associated with neutrophil activation including autocrine interleukin$17 \mathrm{~A}$ production, expression of the upstream transcriptional regulator ROR $\gamma \mathrm{T}$, and oxidative burst potential, ${ }^{24-26}$ each did not shift significantly among Ly6 $\mathrm{G}^{\text {hi }} \mathrm{Ly} 6 \mathrm{C}^{\text {int }}$ neutrophils in commensal bacteria-depleted compared with control mice (Supplementary Figure S2). Similarly, expression of elastase and myeloperoxidase, each associated with extracellular trap formation and neutrophil antifungal activity, ${ }^{27,28}$ were comparable among neutrophils regardless of antibiotic treatment (Figure 3c,d), whereas production of the extracellular trap effector, calprotectin, ${ }^{29}$ was slightly increased among Ly6G ${ }^{\text {hi }}$ Ly6 ${ }^{\text {int }}$ cells from commensal bacteria-depleted mice (Figure 3e). Collectively, these results show the more robust quantitative expansion of neutrophils unleashed by eradication of commensal bacteria does not coincide with obvious shifts in their fungicidal properties on a per-cell basis, and occurs with minimal qualitative shifts in cell activation.

\section{Enhanced antifungal immunity conferred by commensal bacteria eradication requires sustained $L y 6 G^{\text {hi }} L y 6 C^{\text {int }}$ neutrophil expansion}

To more definitively investigate the protective role of $\mathrm{Ly} 6 \mathrm{G}^{\text {hi }} \mathrm{Ly} 6 \mathrm{C}^{\mathrm{int}}$ neutrophils that expand to more enriched levels with commensal bacteria eradication, the impacts of depleting these cells on C. albicans susceptibility were evaluated for antibiotictreated compared with commensal bacteria-replete control mice. As reduced pathogen recovery and improved survival among commensal bacteria-eradicated mice becomes apparent beginning 10 days after infection, with more exaggerated differences in pathogen recovery by day 20 post infection (Figure 1c,d), neutrophil depletion was initiated 10 days after infection and the impacts on pathogen burden enumerated 10 days thereafter. These studies utilized the $1 \mathrm{~A} 8 \mathrm{clone}$ of the antimouse Ly6G antibody that spares $\mathrm{Ly} 6 \mathrm{G}^{\mathrm{lo}} \mathrm{Ly} 6 \mathrm{C}^{\text {hi }}$ inflammatory monocytes, allowing the contribution of $\mathrm{Ly} 6 \mathrm{G}^{\mathrm{hi}} \mathrm{Ly} 6 \mathrm{C}^{\text {int }}$ neutrophils to be evaluated in isolation. ${ }^{30}$ Despite somewhat reduced efficiency of cell depletion compared with that reported after infection with pathogens that cause more acute infection, ${ }^{30}$ the consistent $\sim 50 \%$ depletion of neutrophils from expanded levels in antibiotic-treated mice was still sufficient to override reductions in fungal burden conferred by commensal bacteria eradication (Figure 4a,b). In particular, 20-fold more C. albicans was recovered from antibiotic-treated mice administered anti-Ly6G compared with isotype control antibody (Figure 4b). Comparatively, no significant differences in recoverable fungal pathogen burden were found with antiLy6G antibody among commensal bacteria-replete mice with dampened neutrophil expansion (Figure 4a,b). Interestingly, 

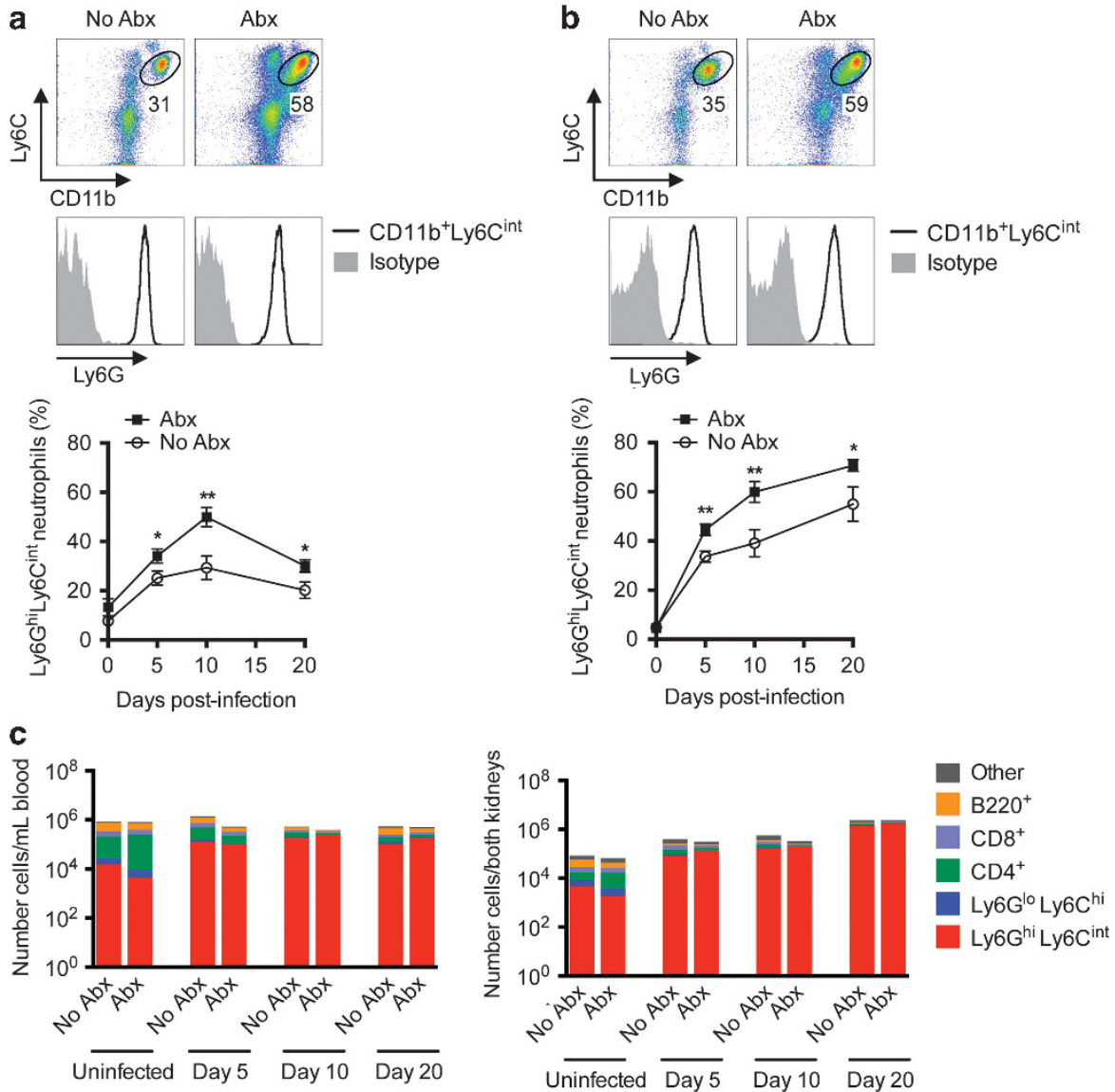

Figure 2 Enriched neutrophil expansion in the blood and kidney for commensal bacteria-depleted mice after intravenous $C$. albicans infection. Representative fluorescence-activated cell sorting plots illustrating the gating scheme (top) and composite data (bottom) for infection-induced shifts in

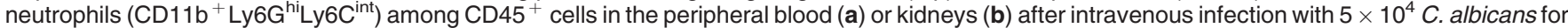
each group of mice. (c) CD45 ${ }^{+}$leukocyte composition in the peripheral blood (left) or kidneys (right) after intravenous $C$. albicans infection. These data are representative of three independent experiments each containing 3-4 mice per group with similar results. ${ }^{\star} P<0.05$, ${ }^{* \star} P<0.01$. Abx, antibiotictreated.

a

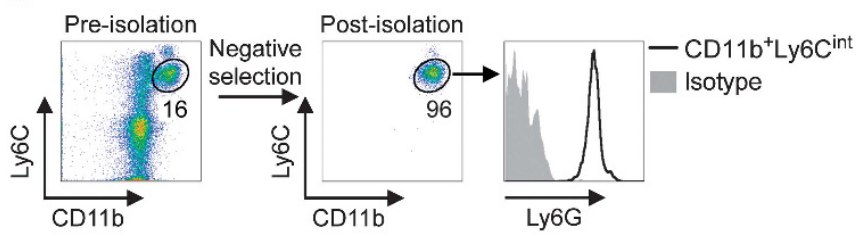

b

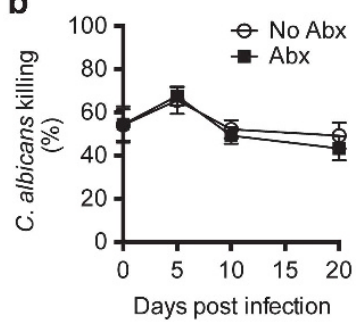

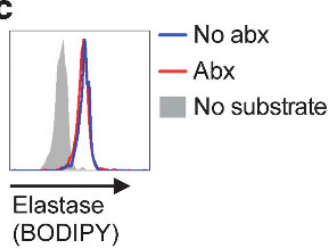

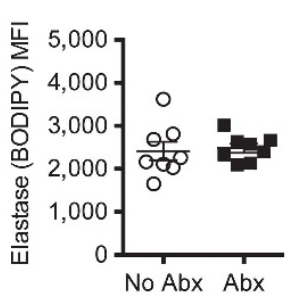

d

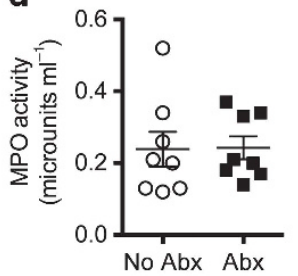

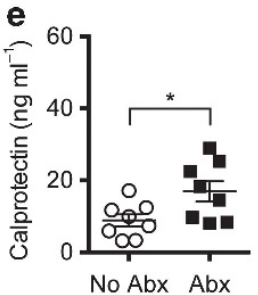

Figure 3 Neutrophil-killing capacity and production of fungicidal molecules among commensal bacteria-depleted mice. (a) Representative fluorescence-activated cell sorting plots illustrating enrichment purity of peripheral blood CD11 $\mathrm{b}^{+}$Ly6G ${ }^{\text {hi }}$ Ly6C ${ }^{\text {int }}$ neutrophils among CD $45^{+}$cells after negative selection. (b) Percent killing by purified CD11b ${ }^{+}$Ly6G ${ }^{\text {hi }}$ Ly6 $C^{\text {int }}$ neutrophils isolated at each time point after intravenous $C$. albicans infection $\left(5 \times 10^{4} \mathrm{CFUs}\right)$, and in vitro incubation with $\mathrm{C}$. albicans at a multiplicity of infection of 0.1 for $60 \mathrm{~min}$ at $37^{\circ} \mathrm{C}$. Representative histogram plots and cumulative mean fluorescence intensity (MFI) of BODIPY dye as a measure of elastase activity (c), myeloperoxidase (MPO) activity (d), and calprotectin production (e) for $5 \times 10^{3} \mathrm{CD} 11 \mathrm{~b}{ }^{+} \mathrm{Ly}_{6 \mathrm{G}}{ }^{\mathrm{hi}} \mathrm{Ly} 6 \mathrm{C}^{\text {int }}$ purified neutrophils isolated from each group of mice 10 days after $C$. albicans infection. These data are representative of at least two independent experiments each with similar results. ${ }^{*} P<0.05$. Abx, antibiotic-treated. 

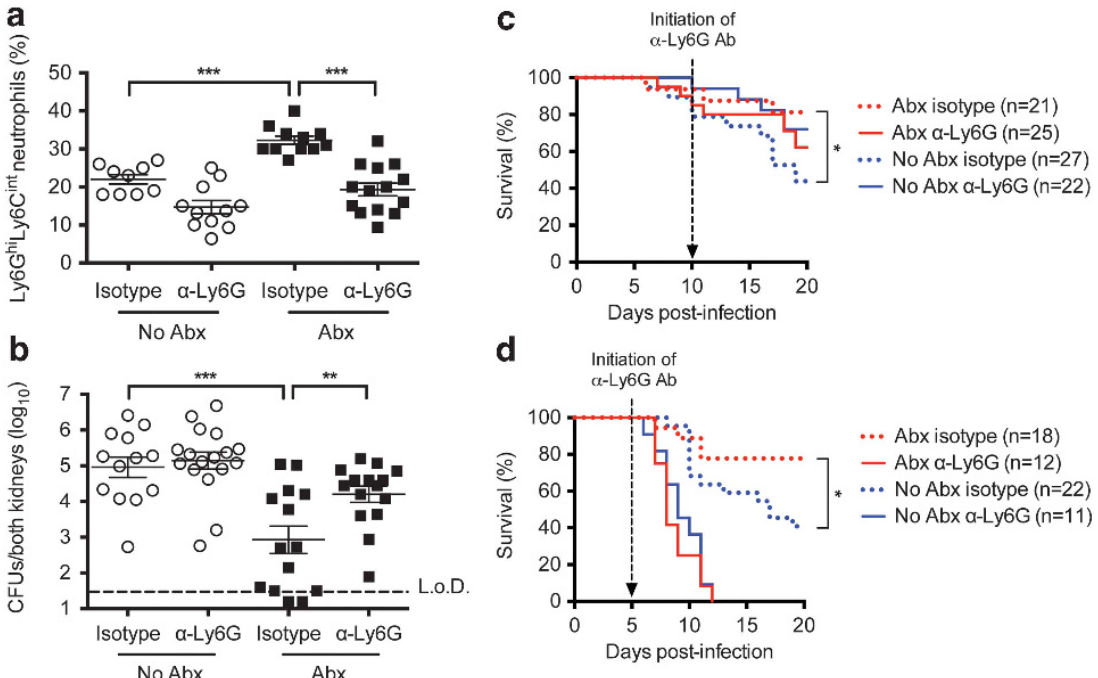

Figure 4 Enhanced protection against disseminated C. albicans infection for commensal bacteria-depleted mice requires sustained Ly6G $\mathrm{G}^{\text {hi }} \mathrm{Ly} 6 \mathrm{C}^{\text {int }}$ neutrophil expansion. (a) Residual CD11 b ${ }^{+}$Ly $6 G^{\text {hi }}{ }^{2 y} 6 C^{\text {int }}$ neutrophils in the peripheral blood 10 days after initiating sustained treatment with anti-Ly6G compared with isotype control antibody for each group of mice day 10 post infection with $5 \times 10^{4} \mathrm{C}$. albicans. Recoverable $\mathrm{C}$. albicans fungal burden day 20 post infection (b) and percent survival (c) among each group of mice described in a. (d) Percent survival after intravenous infection with $5 \times 10^{4}$ C. albicans and initiation of anti-Ly6G compared with isotype control antibody beginning day 5 post infection. These data are representative of three independent experiments each with similar results. ${ }^{\star} P<0.05$, ${ }^{\star *} P<0.01$, ${ }^{* \star *} P<0.001$. Abx, antibiotic-treated; CFUs, colony-forming units; L. o.D., limit of detection.

increased C. albicans pathogen burden after neutrophil depletion among antibiotic-treated mice was not associated with significant differences in overall survival, likely because a substantial proportion of both anti-Ly6G and isotype antibodytreated mice had already begun to succumb by day 10 post infection when neutrophil depletion was initiated (Figure 4c). To circumvent this limitation, related experiments tracked survival for separate groups of mice administered anti-Ly6G compared with isotype control antibody 5 days after intravenous $C$. albicans infection, before any mice became moribund or succumbed to infection. This analysis showed neutrophil depletion initiated earlier after infection eliminated protection conferred by commensal bacteria eradication, as antibiotictreated and control mice each succumbed more rapidly and with similar accelerated kinetics (Figure 4d). Thus, enhanced protection against disseminated C. albicans infection unleashed by eradication of intestinal commensal bacteria requires enriched Ly6G $\mathrm{G}^{\text {hi }} \mathrm{Ly} 6 \mathrm{C}^{\text {int }}$ neutrophil expansion.

\section{Intestinal LPS overrides protection against $C$. albicans conferred by commensal bacteria eradication}

Given the apparent susceptibility to systemic C. albicans infection conferred by commensal intestinal bacteria, we more specifically investigated potential shifts in enteric bacterial density following disseminated fungal infection. As C. albicans can establish colonization in the intestinal lumen following systemic infection, ${ }^{31}$ we first evaluated whether enteric bacteria were retained among mice without antibiotic treatment after C. albicans infection. To discriminate bacteria from C. albicans, microbial growth from the fecal pellet after plating on media without antibiotic supplementation was compared with media supplemented with the same cocktail of antibiotics (ampicillin, gentamicin, metronidazole, neomycin, vancomycin) added to the drinking water. This combination of antibiotics prevents growth of commensal bacteria, and accordingly microbial recovery on antibiotic-supplemented media represent enteric C. albicans, whereas growth on non-supplemented media reflects the cumulative bacterial plus fungal commensal burden. As expected, the feces of mice maintained on drinking water without antibiotic supplementation contained exclusively commensal bacteria, and no fecal organisms could be recovered from antibiotic-treated mice prior to $C$. albicans infection (Figure 5a). Comparatively, after C. albicans intravenous infection, antibiotic-treated mice devoid of commensal bacteria became densely colonized by fungi as comparably high fecal colony-forming units (CFUs) were recovered, regardless of antibiotic supplementation in the culture media (Figure 5a). By contrast, $97 \%$ of the commensal burden in C. albicans-infected mice without antibiotic supplementation in the drinking water was bacterial as the $\sim 10^{6.5} \mathrm{CFUs}^{-1}$ recovered on media without antibiotic supplementation became reduced to only $\sim 10^{5} \mathrm{CFUs} \mathrm{g}^{-1}$ on antibiotic-supplemented media (Figure 5a). Thus, disseminated C. albicans infection does not significantly impact the density of commensal intestinal bacteria among mice without antibiotic treatment.

Considering this drastic difference in commensal intestinal bacteria density among antibiotic-treated compared with control mice, which occurs regardless of $C$. albicans infection, the component of enteric bacteria that confers susceptibility to disseminated fungal infection in commensal bacteria-replete mice was further investigated. Since lipopolysaccharide (LPS) of commensal bacteria has been shown to confer protection against viral pathogens, ${ }^{12}$ we addressed whether this component of enteric bacteria was reciprocally responsible for 


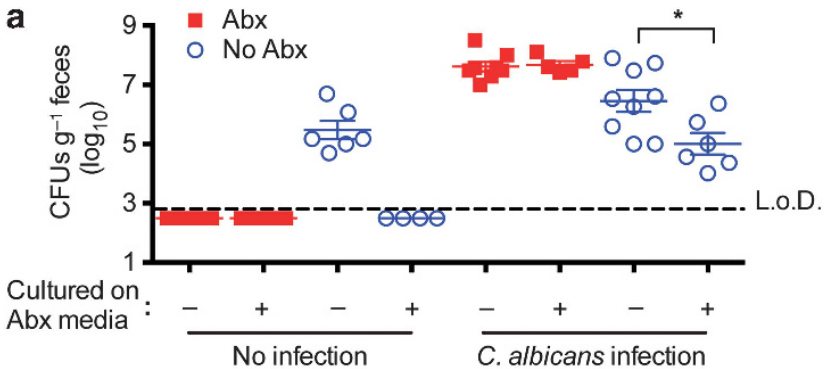

b

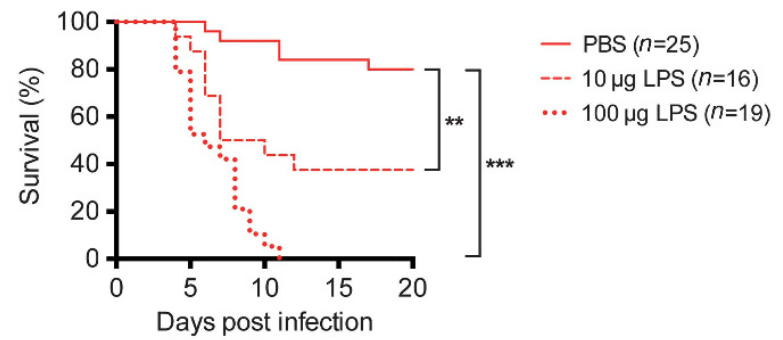

C

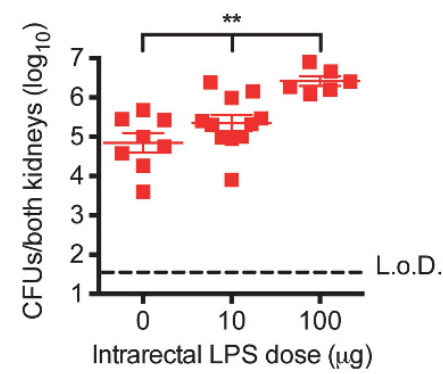

Figure 5 Intestinal lipopolysaccharide (LPS) overrides protection against disseminated $C$. albicans infection conferred by commensal bacteria eradication. (a) Recoverable colony-forming units (CFUs) in fecal pellets for commensal bacteria-depleted or control mice after plating on brain-heart infusion or antibiotic-supplemented media 10 days after intravenous infection with $5 \times 10^{4} \mathrm{C}$. albicans or no infection controls. (b) Percent survival among antibiotic-treated commensal bacteriadepleted mice administered each dosage of intra-rectal LPS or saline controls beginning 2 days prior to and sustained after intravenous infection with $5 \times 10^{4} \mathrm{C}$. albicans. (c) Recoverable $C$. albicans fungal burden day 5 post infection among each group of mice described in $\mathbf{b}$. These data are representative of at least two independent experiments each with similar results. ${ }^{\star} P<0.05,{ }^{\star \star} P<0.01,{ }^{\star \star \star} P<0.001$. Abx, antibiotic-treated; L.o.D., limit of detection, PBS, phosphate-buffered saline.

conferring susceptibility to fungal infection. We found reconstituting mice eradicated of commensal bacteria with LPS restored susceptibility to systemic $C$. albicans infection, as significantly reduced survival and reciprocally increased fungal pathogen burden was recovered with increasing concentrations of intra-rectal LPS administration (Figure 5b,c). Eradication of commensal intestinal bacteria was essential for these detrimental properties of exogenous LPS, as commensal bacteria-replete mice showed no significant differences in survival even at the highest dosage of intra-rectal LPS (Supplementary Figure S3). Taken together, these results demonstrate the LPS component of commensal intestinal bacteria confers susceptibility to disseminated C. albicans infection. a
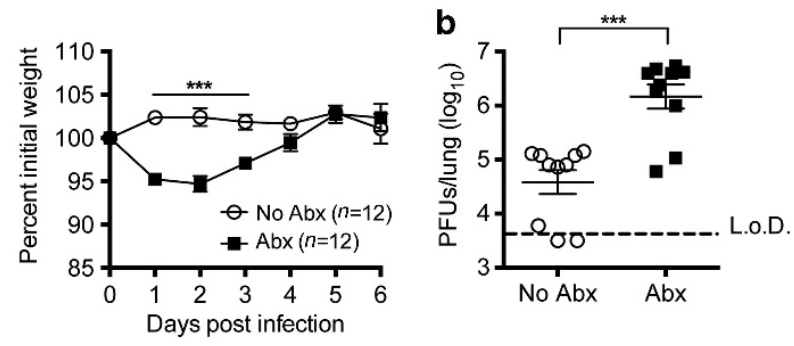

C
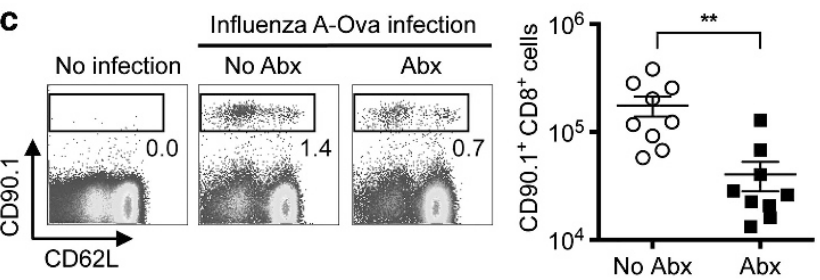

d
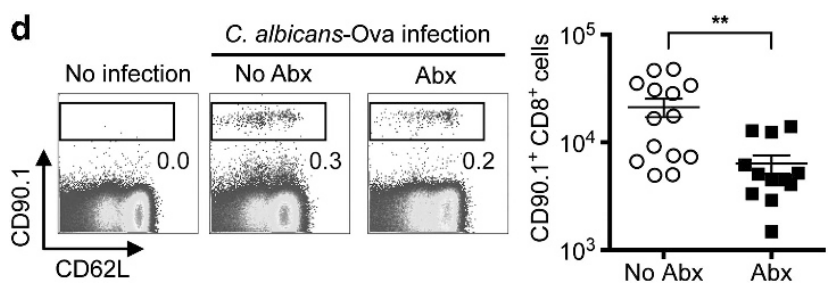

Figure 6 Commensal bacteria eradication blunts pathogen-specific $\mathrm{CD}^{+} \mathrm{T}$-cell expansion after viral and fungal infection. Relative weight loss (a) and recoverable influenza A viral titers (b) day 5 post infection with $10^{3}$ PFUs H1N1 strain PR8-OVA for each group of mice. Representative fluorescence-activated cell sorting plots (left) and composite data (right) showing expansion of OVA-specific CD90.1 ${ }^{+} \mathrm{CD} 8{ }^{+}$cells with surrogate influenza (c) or $C$. albicans (d) specificity among splenocytes day 5 post infection. These data are representative of three independent experiments each with similar results. ${ }^{* \star} P<0.01,{ }^{* * *} P<0.001$. Abx, antibiotic-treated; L.o.D., limit of detection. PFUs, plaque-forming units.

\section{Commensal bacteria promote antiviral immunity and pathogen-specific $\mathrm{CD}^{+}{ }^{+}$-cell expansion regardless of inciting infection}

Additional experiments were performed to investigate the immunological basis for this enhanced antifungal immunity, compared with diminished protection against viral pathogens previously described using the same combination of antibiotics for eradication of commensal intestinal bacteria. ${ }^{11,12}$ First, to address the possibility that differences in commensal bacteria composition between research institutions may influence immune cell responsiveness, ${ }^{32}$ the impacts of commensal bacteria eradication on antiviral immunity after influenza $\mathrm{A}$ virus infection was evaluated in our specific pathogen-free facility. After infection with a sub-lethal dose of recombinant influenza A H1N1, PR8-OVA, which stably expresses the immune-dominant $\mathrm{H}-2 \mathrm{~K}^{\mathrm{b}}$ ovalbumin (OVA) ${ }_{257-264}$ peptide, $^{33}$ significantly more weight loss and recovery of viral plaqueforming units in the lung homogenate were found for antibiotic-treated compared with commensal bacteria-replete control mice (Figure 6a,b). This enhanced susceptibility to influenza A among commensal bacteria-depleted mice paralleled sharp reductions in expansion of OVA-specific $\mathrm{CD}^{+} \mathrm{T}$ cells with surrogate viral specificity (Figure $6 \mathrm{c}$ ). Thus, 
the protective benefits of commensal bacteria on antiviral immunity previously reported using other influenza strains for infection and mice housed at other research facilities are recapitulated. ${ }^{11,12}$

Considering protection against $C$. albicans from enhanced accumulation of neutrophils after eradication of commensal bacteria (Figures 1 and $\mathbf{2}$ ), these results suggest the discordance in antifungal compared with antiviral immunity conferred by commensal intestinal bacteria more likely reflects fundamental differences in how commensal microbes control activation of unique immune components that protect against infection with viral rather than fungal pathogens. To further investigate this hypothesis, the role of commensal intestinal bacteria on expansion of $\mathrm{CD}^{+} \mathrm{T}$ cells after infection with recombinant $C$. albicans, engineered to express the same $\mathrm{H}-2 \mathrm{~K}^{\mathrm{b}}$ $\mathrm{OVA}_{257-264}$ peptide, was evaluated. ${ }^{34}$ Remarkably, a nearidentical reduction in expansion of OVA-specific $\mathrm{CD} 8{ }^{+} \mathrm{T}$ cells among commensal bacteria-depleted mice was observed after infection with recombinant $C$. albicans compared with influenza A (Figure 6c,d). Thus, commensal bacteria uniformly promote antigen-specific $\mathrm{CD} 8^{+} \mathrm{T}$-cell expansion, regardless of the inciting pathogen type, while simultaneously restricting optimal accumulation of Ly $6 \mathrm{G}^{\mathrm{hi}} \mathrm{Ly} 6 \mathrm{C}^{\text {int }}$ neutrophils. Taken together, these results demonstrate that commensal bacteria differentially regulate activation of unique immune cell subsets that, in turn, cause discordant impacts on antifungal compared with antiviral immunity.

\section{DISCUSSION}

Commensal microbes are increasingly recognized to influence responsiveness of immune cells that dictates susceptibility to autoimmunity and infection. As viruses are inherently resistant to antibiotics, shifts in host defense following commensal bacteria elimination have primarily been probed with viral pathogens. Here, commensal bacteria have been shown to have important, but somewhat discordant roles with regard to infection susceptibility. For example, antibiotic-induced eradication of commensal intestinal bacteria consistently impairs host clearance of influenza A virus within the respiratory tract and prolongs viremia after systemic lymphocytic choriomeningitis virus infection. ${ }^{11,12}$ Diminished viral clearance in these instances parallel reduced priming and expansion of protective virus-specific $\mathrm{CD}^{+}{ }^{+} \mathrm{T}$ cells in commensal bacteria-eradicated mice. ${ }^{11,12}$ On the other hand, the presence of commensal intestinal bacteria also promotes susceptibility to numerous enteric viruses including poliovirus, retrovirus, and mouse mammary tumor virus that exploit bacterial LPS to improve transmissibility and infectivity. ${ }^{35,36}$ This latter enhanced capacity for intestinal invasion and replication conferred by commensal bacteria reflects direct viral modulation for enteric viruses, as opposed to systemic shifts in inflammasome or macrophage cell activation that occurs after infection with influenza A or lymphocytic choriomeningitis virus, respectively. ${ }^{11,12}$

Accordingly, to extend the applicability of these findings to systemic immunity against other pathogens that are equally ubiquitous, clinically important, and resistant to antibiotics, the impacts of commensal bacteria eradication on antifungal immunity was investigated using an established murine model of disseminated C. albicans infection. ${ }^{21}$ Surprisingly and in striking contrast to the protective benefits on systemic antiviral immunity, we show commensal bacteria collectively confer detrimental impacts on antifungal host defense. Among antibiotic-treated mice depleted of intestinal commensal bacteria, significantly reduced C. albicans fungal burden and increased overall survival were observed when compared with commensal bacteria-replete control mice. Importantly, these findings cannot be explained by differences in commensal bacteria composition for mice across institutions, considering the antiviral protective benefits of commensal intestinal bacteria previously reported ${ }^{11,12}$ are reproduced after influenza A infection for mice maintained in our facility. Thus, commensal microbes differentially regulate unique immune cell subsets that control antifungal compared with antiviral immunity.

Protection against disseminated C. albicans infection among commensal bacteria-eradicated mice reflects reduced intestinal LPS content, because exogenous intra-rectal LPS administration restored susceptibility in a dose-dependent fashion. When considered in the context of Candida overgrowth at mucosal interfaces after antibiotic administration that may reflect eliminating microbes that compete for limiting local resources, ${ }^{2}$ or commensal bacteria-derived polysaccharides that sustain $\mathrm{CD} 4{ }^{+}$T-cell activation restricting fungal colonization, ${ }^{37,38}$ protection against invasive systemic candidiasis is more specifically mediated by neutrophils. ${ }^{22,23,31}$ These findings may explain why probiotic supplementation in preterm neonates reduces the incidence of gastrointestinal fungal colonization without ameliorating susceptibility to invasive systemic fungal infection. ${ }^{39}$ Reciprocally, neutropenia is consistently identified as a dominant independent risk factor for disseminated candidiasis and fungal infection-induced mortality. ${ }^{40-42}$ Thus, the link between enriched Ly6G $\mathrm{G}^{\mathrm{hi}} \mathrm{Ly} 6 \mathrm{C}^{\mathrm{int}}$ neutrophil expansion and protection against intravenous C. albicans infection we demonstrate using antibiotics to eradicate enteric commensal bacteria further reinforces the dominant role neutrophils have in immunity against systemic candidiasis.

As immune components that mediate antifungal compared with antiviral immunity are largely non-overlapping, this newfound discordance in how commensal bacteria collectively controls host defense against each type of pathogen is perhaps not unexpected. For example, $\mathrm{CD}^{+}{ }^{+} \mathrm{T}$ cells that confer protection against influenza virus and other pathogens that primarily reside within the intracellular compartment of infected host cells have only negligible roles in protection against C. albicans that mainly resides in extracellular compartments. ${ }^{16,17}$ A non-essential role for pathogen-specific $\mathrm{CD}^{+} \mathrm{T}$ cells in host defense against fungi such as C. albicans is consistent with our results, illustrating accelerated fungal clearance despite blunted expansion of $\mathrm{CD}^{+} \mathrm{T}$ cells with surrogate $C$. albicans $\mathrm{OVA}_{257-264}$ specificity among commensal 
bacteria-depleted mice. Instead, we show enhanced antifungal immunity after antibiotic-induced commensal bacteria eradication reflects more robust neutrophil expansion and mobilization, whereas even partial neutrophil depletion with antiLy6G antibody (clone 1A8) overrides these protective benefits. Comparatively among commensal bacteria-replete mice, the detrimental impacts of neutrophil depletion are moderated and potentially reversed as initiating neutrophil depletion 10 days after infection improved survival by $\sim 30 \%$ (although this difference did not reach statistical significance, $P=0.08$ ). These results parallel somewhat paradoxical detrimental roles for neutrophils shown using other loss-of-function approaches for these cells when disseminated candidiasis has already been established. For example, the initiation of neutrophil, together with monocyte and eosinophil, depletion using anti-Gr1 antibody (clone RB6-8C5) 7 days after intravenous C. albicans infection significantly improves overall survival. ${ }^{23,30}$ Similarly, Ccr1-deficient mice with impaired neutrophil accumulation in the kidneys beginning 9 days post infection have improved overall survival after intravenous $C$. albicans infection compared with wild-type control mice. ${ }^{43}$ Thus, although neutrophils are clearly important for protection early after disseminated candidiasis, ${ }^{22,23}$ their accumulation later after fungal infection likely causes immune pathology similar to increased mortality with more robust neutrophil expansion after influenza $\mathrm{A}$ infection. ${ }^{44}$ Taken together, these data suggest commensal bacteria not only control expansion of specific immune cell subsets that dictates whether increased susceptibility or resistance ensues, but shifts in their composition or density may influence the tempo of persistent infection as well.

At first glance our results may also appear contradictory to the clinical association between antibiotic exposure and risk of fungemia with C. albicans and other Candida species. ${ }^{40}$ However, it is also important to highlight the co-existence of other, perhaps, more decisive risk factors including intravascular catheterization, diabetes mellitus, mechanical ventilation, or underlying malignancy that drive the use of antimicrobials among individuals with these immunecompromising conditions, which confers susceptibility to both bacterial and fungal pathogens. ${ }^{42,45}$ Furthermore, our results using orally administered broad-spectrum antibiotics designed to eradicate commensal intestinal bacteria to near completion may not be directly applicable to the clinical use of antibiotics designed to treat or prevent bacterial infections with dysbiosis or disruptions in the composition of commensal bacteria as an inevitable side effect. In particular, the use of antibiotics in most clinical settings almost certainly does not eliminate intestinal bacteria to near completion. Therefore, although our findings attribute impaired antifungal immunity to the presence of bacterial LPS in commensal microbe-replete mice, shifts in the composition of commensal intestinal bacteria likely also have critical roles in modulating susceptibility to fungemia. This notion is supported by the observation that some commensal bacterial species such as segmented filamentous bacteria drive systemic Th17 $\mathrm{CD} 4{ }^{+}$T-cell differentiation ${ }^{32}$ that, in turn, stimulates optimal neutrophil mobilization and immunity against extracellular pathogens including C. albicans. ${ }^{46}$ Reciprocally, this may explain why widely used antibiotics including cephalosporins, penicillins, and vancomycin that each have potent activity against segmented filamentous bacteria are each independently associated with increased risk for candidemia. ${ }^{32,47,48}$ On the basis of these results and the increasing use of antibiotics in clinical medicine, identifying the most essential commensal microbiome subset(s) represents important next steps for dissecting how antifungal compared with antiviral immunity is discordantly regulated.

\section{METHODS}

Mice and adoptive transfers. C57BL/6 mice were purchased from the National Cancer Institute (Bethesda, MD). OT-1 TCR transgenic mice were maintained on a Rag2-deficient C57BL/6 CD90.1 congenic background in our animal facility (Cincinnati, $\mathrm{OH}$ ). For enumerating OVA-specific CD ${ }^{+}$T cells, $2 \times 10^{4} \mathrm{CD}^{+}{ }^{+}$splenocytes from OT-1 transgenic mice maintained on a Rag2-deficient CD90.1 congenic background were adoptively transferred into C57BL/6 (CD90.2 ${ }^{+}$, $\mathrm{CD} 90.1^{-}$) recipient mice 1 day prior to infection. At the indicated time points after infection with recombinant $C$. albicans or recombinant influenza A expressing OVA, CD8 ${ }^{+} \mathrm{T}$ cells with each surrogate pathogen specificity were identified based on CD90.1 $1^{+}$expression. All mice were maintained under specific pathogen-free conditions at the Cincinnati Children's Hospital and used according to institutional animal care and use committee approved protocols.

Antibiotic treatment. The antibiotic cocktail used to eradicate commensal bacteria has been described. ${ }^{11}$ Briefly, drinking water was supplemented with ampicillin $\left(0.5 \mathrm{mg} \mathrm{ml}^{-1}\right.$, Sigma, St Louis, MO), gentamicin $\left(0.5 \mathrm{mg} \mathrm{ml}^{-1}\right.$, Sigma $)$, metronidazole $\left(0.5 \mathrm{mg} \mathrm{ml}^{-1}\right.$ Sigma), neomycin $\left(0.5 \mathrm{mg} \mathrm{ml}^{-1}\right.$, Sigma), vancomycin $\left(0.25 \mathrm{mg} \mathrm{ml}^{-1}\right.$, MP Biomedicals, Solon, OH), and suraclose ( $4 \mathrm{mg} \mathrm{ml}^{-1}$, Sigma). This antibiotic supplementation was initiated $2-3$ weeks prior to infection and continued for the duration of each experiment.

C. albicans and influenza A infection. The recombinant C. albicans SC5314 strain expressing OVA $_{257-264}$ has been described. ${ }^{34}$ For infection, C. albicans was cultured the day prior in yeast extractpeptone-adenine-dextrose media at $30^{\circ} \mathrm{C}(200$ r.p.m. $)$. The following day, the stationary phase culture was diluted in fresh YPAD media for $2 \mathrm{~h}$ to reach exponential phase growth, and the fungi washed and resuspended in sterile saline. Age- and sex-matched C57BL/6 mice were given an intravenous tail-vein injection of $5 \times 10^{4} \mathrm{CFUs}$ (in $200 \mu \mathrm{l}$ phosphate-buffered saline). Influenza A virus expressing the same OVA $_{257-264}$ peptide (PR8-OVA) was diluted to $3.3 \times$ $10^{4} \mathrm{PFUs} \mathrm{ml}^{-1}$, and $30 \mu \mathrm{l}$ was administered intra-nasally to mice anesthetized with xylazine and ketamine. ${ }^{33}$

Recoverable fungal burden and lung viral titers. At the indicated time points after C. albicans inoculation, the kidneys, spleen, liver, and brain were sterilely dissected, homogenized, and serial dilutions of each organ homogenate spread onto agar plates. After incubation for $24 \mathrm{~h}$ at $37^{\circ} \mathrm{C}$, fungal CFUs were enumerated. After influenza A infection, the lungs were collected, homogenized, and viral titers enumerated by counting viral plaques formed from serial dilutions of the lung homogenate plated onto confluent Madin-Darby Canine Kidney cell monolayers. ${ }^{17}$

Tissue collection, flow cytometry and cell depletion. Kidneys were minced into $\sim 1-\mathrm{mm}^{3}$ pieces and then digested with $0.5 \mathrm{mg} \mathrm{ml}^{-1}$ Collagenase D (Sigma), $0.5 \mathrm{mg} \mathrm{ml}^{-1}$ DNAse (Sigma), and $1 \mathrm{mg} \mathrm{ml}^{-1}$ Dispase (Stemcell Technologies, Vancouver, BC) for $45 \mathrm{~min}$ at $37^{\circ} \mathrm{C}$ (200 r.p.m.). Thereafter, the solution was filtered through a $100-\mu \mathrm{m}$ mesh, and the supernatant was pelleted (1,600 r.p.m., 5 min at $4{ }^{\circ} \mathrm{C}$ ), enriched through a percoll gradient (40-80\%), and kidney leukocytes 
were collected and analyzed by fluorescence-activated cell sorting. Blood obtained from the retro-orbital sinus was immediately placed in heparinized tubes and washed with phosphate-buffered saline followed by red blood cell lysis buffer $\left(10 \mathrm{~mm} \mathrm{KHCO}_{3}, 16 \mathrm{~mm} \mathrm{NH}_{4} \mathrm{Cl}, \mathrm{pH}\right.$ 7.3) prior to fluorescence-activated cell sorting analysis. Fluorophoreconjugated antibodies used for flow cytometry include antimouse B220 (RA3-6B2, BioLegend, San Diego, CA), CD4 (GK1.5, eBioscience, San Diego, CA), CD8 (53-6.7, eBioscience), CD11b (M1/70, BioLegend), CD45 (30-F11, eBioscience), Foxp3 (FJK16S, eBioscience), IL-17A (eBio17B7, eBioscience), Ly6C (HK1.4, eBioscience), Ly6G (1A8, BD Biosciences), Ly6G (RB6-8C5, eBioscience), ROR $\gamma \mathrm{T}$ (AFKJS-9, eBioscience). For neutrophil depletion, $500 \mu \mathrm{g}$ of purified anti-Ly6G (1A8, BioXcell, West Lebanon, $\mathrm{NH}$ ) or isotype (Rat IgG2a, BioXcell) antibody was administered by intraperitoneal injection 10 days after $C$. albicans infection with sustained dosing every 3 days thereafter.

Neutrophil isolation and functional assays. $\mathrm{CD} 11 \mathrm{~b}^{+} \mathrm{Ly} 6 \mathrm{G}^{\mathrm{hi}} \mathrm{Ly} 6 \mathrm{C}^{\text {int }}$ neutrophils were isolated from the peripheral blood with negative selection using magnetic bead cell isolation kits (Miltenyi Biotec, San Diego, CA). The purity of CD $11 b^{+}$Ly $6 G^{\text {hi }}{ }^{\text {Ly } 6 C^{\text {int }}}$ cells was verified to be $>95 \%$ by fluorescence-activated cell sorting analysis. To quantify myeloperoxidase activity, $5 \times 10^{3}$ neutrophils were seeded into 96-well plates and incubated with aminophenyl fluorescein (Sigma) for $2-3 \mathrm{~h}$ at room temperature. Production of the fluorescent product, fluorescein, reflective of myeloperoxidase activity was measured using a plate reader ( $485 \mathrm{~nm}$ excitation, $535 \mathrm{~nm}$ emission). The elastase assay was performed by incubating $5 \times 10^{3}$ purified neutrophils with BODIPY-conjugated elastin (Life Technologies, Grand Island, NY) for $30 \mathrm{~min}$ at $37^{\circ} \mathrm{C}$. Thereafter, the fluorescence intensity of the elastase product, BODIPY, was measured by flow cytometry. To measure calprotectin secretion, $5 \times 10^{3}$ purified neutrophils were resuspended in Dulbecco's modified Eagle's medium (Life Technologies) and incubated for $30 \mathrm{~min}$ at $37^{\circ} \mathrm{C}$. Thereafter, the supernatant was collected and analyzed by enzyme-linked immunosorbant assay according to the manufacturer's instructions (Hycult Biotech, Plymouth Meeting, PA). The in vitro neutrophil-killing assay was performed as previously described. ${ }^{49}$ Briefly, peripheral blood neutrophils were purified and $5 \times 10^{3}$ neutrophils were incubated with $5 \times 10^{2} \mathrm{CFU}$ C. albicans (multiplicity of infection of 0.1 ) for $1 \mathrm{~h}$ at $37^{\circ} \mathrm{C}$ with gentle agitation in 96-well round-bottom plates. Thereafter, C. albicans CFUs were enumerated after spreading serial dilutions of the co-culture mixture on agar plates. The percent killing of Candida was defined as $(1-$ (CFU after incubation/CFU recovered at start of incubation) $) \times 100$. For the dihydrorhodamine 123 assay, leukocytes were seeded into 96-well round-bottom plates with media supplemented with dihydrorhodamine 123 (final concentration $1 \mu \mathrm{M}$ per well), and incubated for $30 \mathrm{~min}$ at $37^{\circ} \mathrm{C}$. Thereafter, the fluorescence intensity of rhodamine 123 illustrating oxidative burst potential was measured by flow cytometry. ${ }^{50}$

Rectal LPS inoculation. Mice anesthetized with xylazine and ketamine were intra-rectally administered the indicated dosage of ultrapure Escherichia coli LPS (InvivoGen, San Diego, CA) resuspended in $100 \mu \mathrm{l}$ sterile saline, as described in Ichinohe et al., ${ }^{12}$ beginning 2 days prior to $C$. albicans infection with repeated dosing every 2 days thereafter.

Statistical analysis. Differences in survival were compared using the log rank (Mantel-Cox) test. Cell frequencies, enzymatic activity, and protein secretion were analyzed with the unpaired Student's $t$-test, and differences in pathogen burden were compared with the non-parametric Mann-Whitney test (two groups) or non-parametric KruskalWallis one-way analysis of variance ( $>2$ groups). In all cases, $P<0.05$ was taken as the statistical significance.

SUPPLEMENTARY MATERIAL is linked to the online version of the paper at http://www.nature.com/mi

\section{ACKNOWLEDGMENTS}

We thank Daniel Kaplan (University of Minnesota) for providing C. albicans, Thomas Moran (Ichan School of Medicine at Mount Sinai), and Paul Thomas (Saint Jude Children's Research Hospital) for providing PR8-OVA influenza A virus. This research was supported in part by the NIH and NIAID under awards (R01Al100934, R01Al087830, and R21Al112186) and the National Institute of General Medical Sciences (T32-GM063483). S.S.W. holds an Investigator in the Pathogenesis of Infectious Disease award from the Burroughs Wellcome Fund.

\section{DISCLOSURE}

The authors declared no conflict of interest.

(c) 2015 Society for Mucosal Immunology

\section{REFERENCES}

1. Maynard, C.L., Elson, C.O., Hatton, R.D. \& Weaver, C.T. Reciprocal interactions of the intestinal microbiota and immune system. Nature 489, 231-241 (2012).

2. Högenauer, C., Hammer, H.F., Krejs, G.J. \& Reisinger, C. Mechanisms and management of antibiotic-associated diarrhea. Clin. Infect. Dis. 27, 702-710 (1998).

3. Manichanh, C., Borruel, N., Casellas, F. \& Guarner, F. The gut microbiota in IBD. Nat. Rev. Gastroenterol. Hepatol. 9, 599-608 (2012).

4. Garrett, W.S. et al. Communicable ulcerative colitis induced by T-bet deficiency in the innate immune system. Cell 131, 33-45 (2007).

5. Clarke, T.B. et al. Recognition of peptidoglycan from the microbiota by Nod1 enhances systemic innate immunity. Nat. Med. 16, 228-231 (2010).

6. Turnbaugh, P.J. et al. An obesity-associated gut microbiome with increased capacity for energy harvest. Nature 444, 1027-1131 (2006).

7. Wen, L. et al. Innate immunity and intestinal microbiota in the development of Type 1 diabetes. Nature 455, 1109-1113 (2008).

8. Kriegel, M.A. et al. Naturally transmitted segmented filamentous bacteria segregate with diabetes protection in nonobese diabetic mice. Proc. Natl. Acad. Sci. USA 108, 11548-11553 (2011).

9. Wu, H.-J. et al. Gut-residing segmented filamentous bacteria drive autoimmune arthritis via T helper 17 cells. Immunity 32, 815-827 (2010).

10. Lee, Y.K., Menezes, J.S., Umesaki, Y. \& Mazmanian, S.K. Proinflammatory T-cell responses to gut microbiota promote experimental autoimmune encephalomyelitis. Proc. Natl. Acad. Sci. USA 108, 4615-4622 (2011).

11. Abt, M.C. et al. Commensal bacteria calibrate the activation threshold of innate antiviral immunity. Immunity 37, 158-170 (2012).

12. Ichinohe, T. et al. Microbiota regulates immune defense against respiratory tract influenza A virus infection. Proc. Natl. Acad. Sci. USA 108, 5354-5359 (2011).

13. Gagneur, A. et al. Coronavirus-related nosocomial viral respiratory infections in a neonatal and paediatric intensive care unit: a prospective study. J. Hosp. Infect. 51, 59-64 (2002).

14. Szajewska, H. \& Mrukowicz, J.Z. Probiotics in the treatment and prevention of acute infectious diarrhea in infants and children: a systematic review of published randomized, double-blind, placebo-controlled trials. J. Pediatr. Gastroenterol. Nutr. 33, S17-S25 (2001).

15. Qian, Q. \& Cutler, J.E. Gamma interferon is not essential in host defense against disseminated candidiasis in mice. Infect. Immun. 65, 1748-1753 (1997).

16. Balish, E., Vazquez-Torres, F.A., Jones-Carson, J., Wagner, R.D. \& Warner, T. Importance of beta2-microglobulin in murine resistance to mucosal and systemic candidiasis. Infect. Immun. 64, 5092-5097 (1996).

17. Brincks, E.L., Katewa, A., Kucaba, T.A., Griffith, T.S. \& Legge, K.L. CD8 T cells utilize TRAIL to control influenza virus infection. J. Immunol. 181, 4918-4925 (2008).

18. Majer, O. et al. Type I interferons promote fatal immunopathology by regulating inflammatory monocytes and neutrophils during Candida infections. PLoS Pathog. 8, e1002811 (2012).

19. del Fresno, C. et al. Interferon- $\beta$ production via Dectin-1-Syk-IRF5 signaling in dendritic cells is crucial for immunity to $C$. albicans. Immunity 38, 1176-1186 (2013) 
20. Huang, W., Na, L., Fidel, P.L. \& Schwarzenberger, P. Requirement of interleukin-17A for systemic anti-Candida albicans host defense in mice. J. Infect. Dis. 190, 624-631 (2004).

21. MacCallum, D.M. \& Odds, F.C. Temporal events in the intravenous challenge model for experimental Candida albicans infections in female mice. Mycoses 48, 151-161 (2005).

22. Fulurija, A., Ashman, R.B. \& Papadimitriou, J.M. Neutrophil depletion increases susceptibility to systemic and vaginal candidiasis in mice, and reveals differences between brain and kidney in mechanisms of host resistance. Microbiology 142, 3487-3496 (1996).

23. Romani, L. et al. An immunoregulatory role for neutrophils in CD4+ $\mathrm{T}$ helper subset selection in mice with candidiasis. J. Immunol. 158, 2356-2362 (1997).

24. Taylor, P.R. et al. Activation of neutrophils by autocrine IL-17A-IL-17RC interactions during fungal infection is regulated by IL-6, IL-23, ROR $\gamma \mathrm{t}$ and dectin-2. Nat. Immunol. 15, 143-151 (2014).

25. Werner, J.L. et al. Neutrophils produce interleukin 17A (IL-17A) in a dectin1-and IL-23-dependent manner during invasive fungal infection. Infect. Immun. 79, 3966-3977 (2011).

26. Diamond, R.D., Lyman, C.A. \& Wysong, D.R. Disparate effects of interferon-gamma and tumor necrosis factor-alpha on early neutrophil respiratory burst and fungicidal responses to Candida albicans hyphae in vitro. J. Clin. Invest. 87, 711 (1991).

27. Urban, C.F., Reichard, U., Brinkmann, V. \& Zychlinsky, A. Neutrophil extracellular traps capture and kill Candida albicans yeast and hyphal forms. Cell. Microbiol. 8, 668-676 (2006).

28. Papayannopoulos, V., Metzler, K.D., Hakkim, A. \& Zychlinsky, A. Neutrophil elastase and myeloperoxidase regulate the formation of neutrophi extracellular traps. J. Cell Biol. 191, 677-691 (2010).

29. Urban, C.F. et al. Neutrophil extracellular traps contain calprotectin, a cytosolic protein complex involved in host defense against Candida albicans. PLoS Pathog. 5, e1000639 (2009).

30. Shi, C. et al. Ly6G + neutrophils are dispensable for defense against systemic Listeria monocytogenes infection. J. Immunol. 187, 5293-5298 (2011).

31. Koh, A.Y., Köhler, J.R., Coggshall, K.T., Van Rooijen, N. \& Pier, G.B. Mucosal damage and neutropenia are required for Candida albicans dissemination. PLoS Pathog. 4, e35 (2008).

32. Ivanov, I.I. et al. Induction of intestinal Th17 cells by segmented filamentous bacteria. Cell 139, 485-498 (2009).

33. Jenkins, M.R., Webby, R., Doherty, P.C. \& Turner, S.J. Addition of a prominent epitope affects influenza A virus-specific CD8 $+\mathrm{T}$ cell immunodominance hierarchies when antigen is limiting. J. Immunol. 177, 2917-2925 (2006).

34. Igyártó, B.Z. et al. Skin-resident murine dendritic cell subsets promote distinct and opposing antigen-specific T helper cell responses. Immunity 35, 260-272 (2011).
35. Kane, M. et al. Successful transmission of a retrovirus depends on the commensal microbiota. Science 334, 245-249 (2011).

36. Kuss, S.K. et al. Intestinal microbiota promote enteric virus replication and systemic pathogenesis. Science 334, 249-252 (2011).

37. Conti, H.R. et al. Th17 cells and IL-17 receptor signaling are essential for mucosal host defense against oral candidiasis. J. Exp. Med. 206, 299-311 (2009).

38. Mazmanian, S.K., Liu, C.H., Tzianabos, A.O. \& Kasper, D.L. An immunomodulatory molecule of symbiotic bacteria directs maturation of the host immune system. Cell 122, 107-118 (2005).

39. Manzoni, P. et al. Oral supplementation with Lactobacillus casei subspecies rhamnosus prevents enteric colonization by Candida species in preterm neonates: a randomized study. Clin. Infect. Dis. 42, 1735-1742 (2006).

40. Viudes, A. et al. Candidemia at a tertiary-care hospital: epidemiology, treatment, clinical outcome and risk factors for death. Eur. J. Clin. Microbiol. Infect. Dis. 21, 767-774 (2002).

41. Abi-Said, D. et al. The epidemiology of hematogenous candidiasis caused by different Candida species. Clin. Infect. Dis. 24, 1122-1128 (1997).

42. Pappas, P.G. et al. A prospective observational study of candidemia: epidemiology, therapy, and influences on mortality in hospitalized adult and pediatric patients. Clin. Infect. Dis. 37, 634-643 (2003).

43. Lionakis, M.S. et al. Chemokine receptor Ccr1 drives neutrophil-mediated kidney immunopathology and mortality in invasive candidiasis. PLOS Pathog. 8, e1002865 (2012).

44. Brandes, M., Klauschen, F., Kuchen, S. \& Germain, R.N. A systems analysis identifies a feedforward inflammatory circuit leading to lethal influenza infection. Cell 154, 197-212 (2013).

45. Horn, D.L. et al. Epidemiology and outcomes of candidemia in 2019 patients: data from the prospective antifungal therapy alliance registry. Clin. Infect. Dis. 48, 1695-1703 (2009).

46. Curtis, M.M. \& Way, S.S. Interleukin-17 in host defence against bacterial, mycobacterial and fungal pathogens. Immunology 126, 177-185 (2009).

47. Karabinis, A. et al. Risk factors for candidemia in cancer patients: a casecontrol study. J. Clin. Microbiol. 26, 429-432 (1988).

48. Klaasen, $\mathrm{H}$. et al. Influence of antimicrobial drugs on segmented filamentous bacteria in the ileum of mice. Microb. Ecol. Health Dis. 4, 391-397 (1991).

49. Netea, M.G. et al. Increased susceptibility of TNF- $\alpha$ lymphotoxin- $\alpha$ double knockout mice to systemic candidiasis through impaired recruitment of neutrophils and phagocytosis of Candida albicans. J. Immunol. 163, 1498-1505 (1999).

50. Rothe, G., Oser, A. \& Valet, G. Dihydrorhodamine 123: a new flow cytometric indicator for respiratory burst activity in neutrophil granulocytes. Naturwissenschaften 75, 354-355 (1988). 